\title{
Nano- scale X-ray Computed Tomography: Morphological Analysis of Fuel Cell Electrodes
}

\author{
Pratiti Mandal, William K. Epting, and Shawn Litster \\ Carnegie Mellon University, Mechanical Engineering Department, Pittsburgh, PA, USA
}

We report on the use of nano-scale X-ray Computed Tomography (nano-CT) to study the structural features of polymer electrolyte fuel cell (PEFC) electrodes, with the aim of relating the structure to transport phenomena. PEFC-powered automobiles are a promising technology for reducing the pollution attributed to the transportation sector. However, PEFC costs must be reduced before the technology is widely competitive. The PEFC's catalyst layer has a composite porous structure consisting of platinum and carbon nanoparticles in a polymer electrolyte binder; these aggregate together to form discrete agglomerates, which in turn assemble together to form the porous structure of the catalyst layer. Our study mainly focuses on the cathode material because of the high loadings of costly Pt catalyst required for oxygen reduction in order to achieve high PEFC performance.

Here, we use X-ray nano-CT, a non-invasive and non-destructive method of obtaining high resolution 3D images of complex structures. High resolution is obtained by using advanced optics, including a single capillary condenser and a Fresnel zone plate objective. A schematic of the X-ray optics is shown in Figure 1. The nano-CT (UltraXRM-L200, Xradia Inc., Pleasanton, CA, USA) at Carnegie Mellon is a lab scale instrument that allows a maximum resolution of $50 \mathrm{~nm}$ with a $16.5 \mu \mathrm{m}$ field of view. With the use of Zernike phase contrast, this system can achieve its high resolutions on materials of low atomic number; i.e. materials with low absorption contrast, such as organic materials, polymers, and carbon found in PEFC electrodes.

Our previous reports on using nano-CT have included imaging of PEFC catalyst layers and subsequent quantification of porosity and pore and solid phase size distributions [1]. We also used nano-CT data to evaluate the effects of an agglomerate size distribution on the predictions of a commonly used agglomerate model for the catalyst layer [2]. In the present study, we extend the previous work by imaging the individual agglomerates present in the catalyst ink used for making the catalyst layer (Figure 2a), sparsely dispersed on commercially available low X-ray attenuating polymer films like polyimide (Kapton $\left.{ }^{\circledR}\right)$ or polyethylene terephthalate (Mylar ${ }^{\circledR}$ ). By isolating the constituent agglomerates, the computed tomography of these sub-micron sized agglomerates yields further insight into the electrode morphology. Additionally, we have imaged a new catalyst layer sample (Figure 2b) and analyzed the results using custom codes and commercial software (Avizo ${ }^{\circledR}$ Fire, VSG, Burlington, MA, USA). We thus calculated the agglomerate size distribution in the catalyst layer and compared them with the dispersed agglomerates. Together, the images of the catalyst layer and dispersed agglomerates yield a more complete picture of the effect of catalyst layer fabrication techniques on the resulting structure. Moreover, the resulting quantified morphological parameters can be an input for modeling pore- and solid-phase transport phenomena, providing further insight into the effect of electrode structure on a PEFC's electrochemical performance [3]. 


\section{References:}

[1] W. K. Epting, J. Gelb and S. Litster, Advanced Functional Materials 22, (2012), p. 555.

[2] W. K. Epting and S. Litster, International Journal of Hydrogen Energy, 37, (2012), p. 8505.

[3] The authors gratefully acknowledge the National Science Foundation under MRI Grant No. 1229090 in support of the nano-CT acquisition.

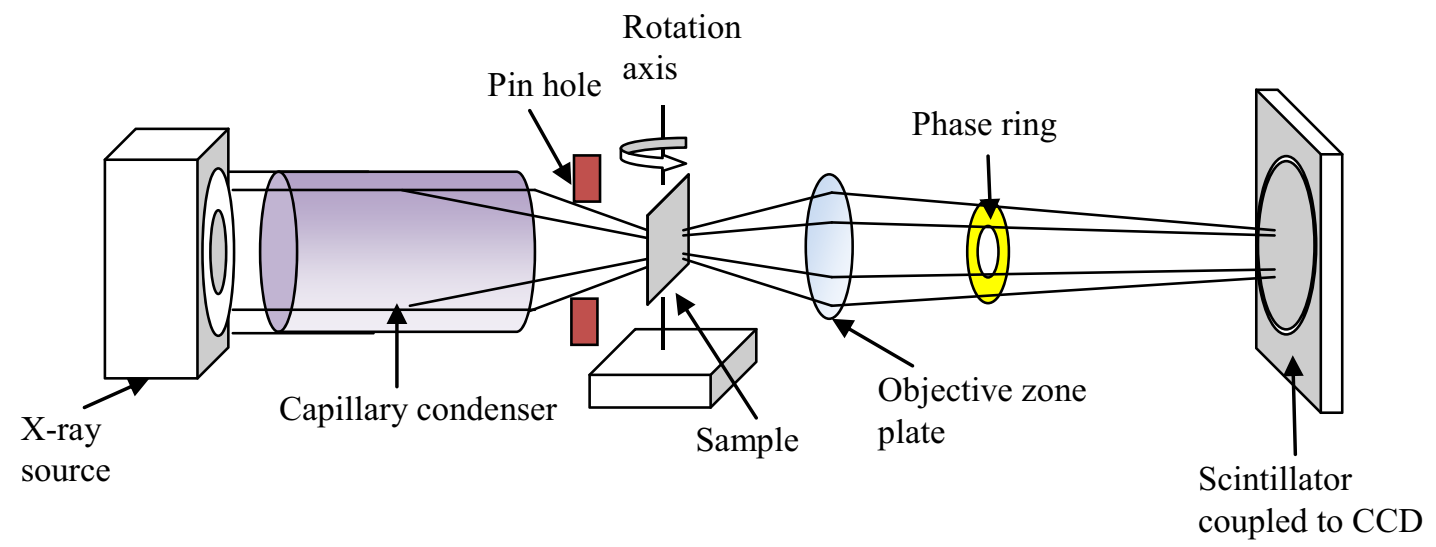

Figure 1. Schematic of X-ray optics in the UltraXRM-L200

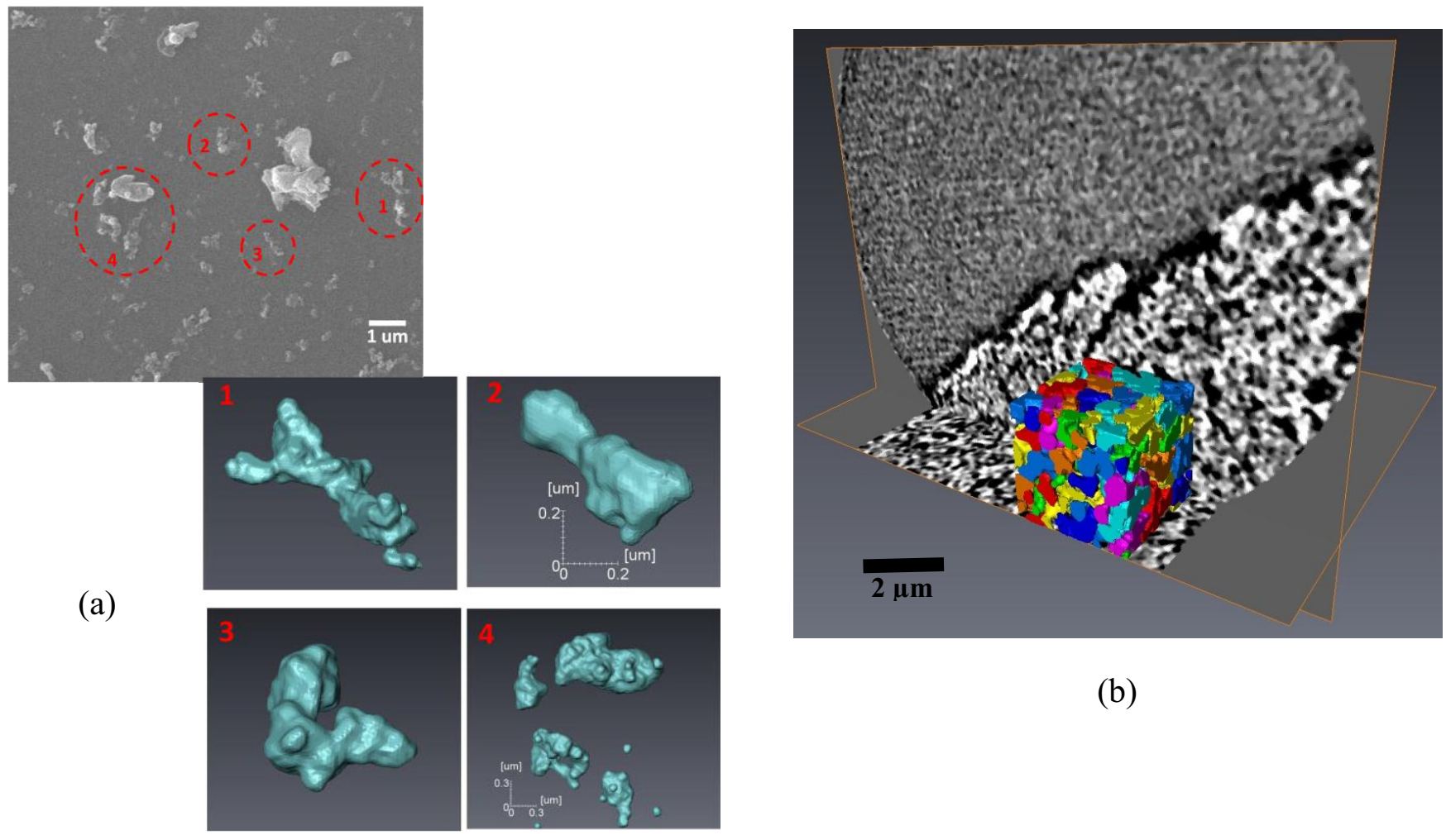

Figure 2. The morphology of a PEFC cathode catalyst layer. (a) SEM (top-left) and nano-CT (bottomright) images of the same isolated agglomerates of dispersed catalyst layer material. (b) Color coded agglomerate structures comprising the solid phase of a porous catalyst layer fabricated from the same materials as in (a). 